\title{
Expectations for detection of neutrinos from point-like sources with KM3NeT/ARCA
}

\section{A. Trovato*}

INFN, Laboratori Nazionali del Sud, Via S. Sofia 62, Catania, 95123 Italy

E-mail: atrovato@lns.infn.it

\section{R. Coniglione ${ }^{\dagger}$}

INFN, Laboratori Nazionali del Sud, Via S. Sofia 62, Catania, 95123 Italy

E-mail: coniglionedlns.infn.it

\section{P. Sapienza}

INFN, Laboratori Nazionali del Sud, Via S. Sofia 62, Catania, 95123 Italy

E-mail: sapienza@lns.infn.it

\section{J. Barrios-Martí}

IInstituto de Física Corpuscular, IFIC (UV-CSIC), Parque Científico, C/Catedrático José

Beltrán 2, E-46980 Paterna, Spain

Email:jabamar@ific.uv.es

\section{for the KM3NeT Collaboration}

The KM3NeT Collaboration has started the implementation of the first phase of the ARCA cubickilometre-scale neutrino telescope in the Northern hemisphere integrated in a platform for Earth and deep sea sciences. The ARCA detector will be located in the Mediterranean Sea close to the Sicilian coast, about $100 \mathrm{~km}$ offshore Capo Passero at a depth of $3500 \mathrm{~m}$. This location will allow surveying a large part of the sky, including most of the Galactic Plane and the Galactic Centre. Theoretical models for neutrino emission, that predict neutrino fluxes from gamma-ray and cosmic-ray measurements, can be robustly constrained by the neutrino observation with a detector with an excellent pointing accuracy such as ARCA. In the KM3NeT Letter of Intent the first estimates of the discovery fluxes from benchmark fluxes from candidate Galactic neutrino sources have been reported together with the discovery flux for generic $E^{-2}$ point-like sources. A more sophisticated analysis has been developed by the KM3NeT Collaboration and new expectations will be shown not only for the supernova remnant RXJ1713.7-3946 and the pulsar wind nebula Vela $\mathrm{X}$ but also for other potential neutrino sources in our Galaxy. Expectations for a generic point-like source with a spectrum proportional to $E^{-2}$ will be reported.

35th International Cosmic Ray Conference - ICRC2017

10-20 July, 2017

Bexco, Busan, Korea

\footnotetext{
* Corresponding author

${ }^{\dagger}$ Speaker.
} 


\section{Introduction}

KM3NeT [1] is a $\mathrm{km}^{3}$-scale high-energy neutrino telescope being installed in the Mediterranean Sea. The discovery by the IceCube experiment of a high-energy neutrino flux of extraterrestrial origin [2] and the $\theta_{13}$ measurement motivated the Collaboration to propose KM3NeT-2.0 [3] consisting of two detectors with different granularity, KM3NeT/ARCA and KM3NeT/ORCA (Astroparticle and Oscillation Research in the Abyss). ARCA is dedicated to high-energy neutrino astronomy and is being installed at the KM3NeT-It site off-shore Capo Passero (Italy). With ORCA the aim is to study the neutrino mass hierarchy. ORCA is being installed at the KM3NeT-Fr site offshore Toulon (France). ORCA will have a Mton scale and it will be sensitive to $\mathrm{GeV}$ energies while for KM3NeT/ARCA the size will be of Gton scale and the energy threshold around 1-10 $\mathrm{TeV}$. This paper will focus on the scientific potential of the ARCA detector.

Neutrinos are an unique probe to observe high energy astrophysical phenomena, since they interact only weakly with matter and are not subject to the influence of magnetic fields. Therefore, they can travel long distances without being deflected or absorbed and can provide unique information on the most violent and highest energy processes in our Galaxy and far beyond. At the moment our knowledge of the high energy Universe is based on measurements of charged cosmic rays (CRs) and $\gamma$-rays. The trajectory of charged CRs their trajectory is bent by galactic and extragalactic magnetic fields and consequently do not point back at the sources. Photons, like neutrinos, are produced in the astrophysical beam dump of the primary cosmic rays particles interacting with the ambient matter at the source location. The origin of CRs is still uncertain. In our Galaxy the standard paradigm foresee that the Galactic CRs are accelerated to high energy in young supernova remnants (SNRs), that are expanding shock waves following the supernova explosions [4]. In the last decades a large number of Galactic young SNRs have been identified by $\gamma$-ray telescopes. The observed $\gamma$-ray spectrum extends up to tens of $\mathrm{TeV}$ proving that these objects are accelerators of high energy particles. These particles could be protons that emit $\gamma$-rays via inelastic production of neutral pions, but could also be electrons which emit very high-energy $(E>100 \mathrm{GeV}) \gamma$-rays via Inverse Compton scattering of ambient low energy photons. The unequivocal proof of the hadronic mechanism is difficult to achieve with $\gamma$-rays observations only, while neutrinos observations would be a smoking gun signature of cosmic rays acceleration, since neutrinos can only be emitted from the decay of charged pions produced by protons.

Another class of galactic objects detected in the TeV band are Pulsar Wind Nebulae (PWN), nebulae of non-thermal radiation powered by pulsar winds, namely relativistic magnetized outflows emitted by a highly spinning, strongly magnetized neutron star, usually detected as pulsar. Since the pulsar is created by a supernova explosion, both the pulsar and its wind are initially located within the SNR. The interaction of the pulsar wind with the slower supernova ejecta or with the interstellar medium creates a termination shock where particles can be accelerated to very high energies. The $\mathrm{TeV}$ emission of PWN is usually interpreted in a purely leptonic scenario, but according to some authors a hadronic contribution could be present [5].

In this paper, neutrino fluxes expected from a selected list of galactic sources are estimated assuming a hadronic scenario for the $\gamma$-ray production and transparent sources. The perspective for detection by ARCA of neutrinos from these specific sources is discussed as is ARCA sensitivity to a generic $E^{-2}$ flux. The analysis is similar to what is presented in [3] but has been improved and 
additional sources have been considered.

\section{The KM3NeT/ARCA detector}

Neutrinos can be detected by measuring the Cherenkov light induced by charged secondary particles emerging from a neutrino interaction. KM3NeT will consist of arrays of light detectors deployed in the Italian site of Capo Passero at a depth of $3500 \mathrm{~m}$ in the clear water of the Mediterranean Sea that serves both as target material and Cherenkov radiator and shields against atmospheric muons. The typical energy range of interest of the underwater Cherenkov technique is of the order of magnitude between $\mathrm{TeV}$ and $\mathrm{PeV}$. The light detectors are photo-multiplier tubes (PMTs) arranged in glass spheres that can withstand the water pressure (digital optical modules, DOM [6]). In KM3NeT, the optical modules will house multiple small PMTs instead of the traditional one ( 31 3" PMTs), optimizing the photo-cathode area per optical module. The DOMs are arranged along flexible strings with a total height of about $700 \mathrm{~m}$. The detector will consist of two building blocks of 115 strings each, with 18 DOMs per string, vertically spaced by $36 \mathrm{~m}$. The footprint will be roughly circular with an average distance between strings of about $90 \mathrm{~m}$. The two blocks together will have a volume of about $1 \mathrm{~km}^{3}$.

\section{Simulations}

Monte Carlo (MC) simulations are needed to evaluate the potential of the detector. A detailed description of the simulation programs can be found in [3]. Neutrinos of all flavours with energy in the range $10^{2}-10^{8} \mathrm{GeV}$ are simulated, including their interaction in the medium, the propagation of the emerging secondary particles, the light generation and propagation in water and the detector response. The neutrino induced events are observed in two topologies: track-like and cascade-like events, each class requiring a specific event reconstruction.

Track-like events are due to $\mathrm{CC} v_{\mu}$ and $\bar{v}_{\mu}$ interactions that, for $E_{v} \gtrsim 1 \mathrm{TeV}$, produce in the final state muons with track lengths in the order of kilometers and trajectories almost collinear with the parent neutrino direction. Also $v_{\tau}\left(\bar{v}_{\tau}\right) \mathrm{CC}$ interactions can produce a muon in the final state when the emerging $\tau(\bar{\tau})$ decays in a $\mu(\bar{\mu})(\mathrm{BR} \sim 17 \%)$. The reconstruction algorithm used for track-like events is described in [7]. Initial values of the candidate reconstructed tracks are obtained from a scan of the full solid angle. A likelihood is then maximized using a multi-dimensional probability distribution of the arrival times of the Cherenkov light from the muon. The track with the best likelihood is chosen as final reconstructed track. The angular resolution, calculated as the median angle between the reconstructed track and the generated neutrino track, is about $0.2^{\circ}$ or better at $\mathrm{E}_{v}>10 \mathrm{TeV}$. The energy resolution is better than $30 \%$ in $\log _{10}\left(\mathrm{E}_{\text {reco }} / \mathrm{E}_{\mu}\right)$.

The cascade-like topology is originated from $v_{e}\left(\bar{v}_{e}\right)$ CC interactions, $v_{\tau}\left(\bar{v}_{\tau}\right)$ CC interactions when the emerging $\tau(\bar{\tau})$ produces a shower in the final state (BR $\sim 83 \%)$ and all NC neutrino interactions. The direction reconstruction for cascade-like events is based on the principle that the amount of light emitted under the Cherenkov angle with respect to the shower axis is larger than that in the other directions [7]. For high energy cascades $\left(E_{v}>50 \mathrm{TeV}\right)$ taking place within the instrumented volume, a median angular resolution of less than $2^{\circ}$ and an energy resolution of roughly $5 \%$ are reached. 
Table 1: List of the source declination, extension and neutrino flux and reference of the corresponding $\gamma$-ray flux. The flux is expressed according to equation 4.1 but with the normalization constant $\Phi_{0}$ in units of $10^{-11} \mathrm{TeV}^{-1} \mathrm{~s}^{-1} \mathrm{~cm}^{-2}$. See the text for further details.

\begin{tabular}{llllllll}
\hline Source & $\delta$ & extension & $\Phi_{0}$ & $\Gamma$ & $E_{\text {cut }}$ & $\beta$ & $\gamma$-ray data \\
\hline \hline RX J1713.7-3946 (1) & $-39.77^{\circ}$ & $0.6^{\circ}$ & 1.68 & 1.72 & 2.1 & 0.5 & {$[10]$} \\
\hline RX J1713.7-3946 (2) & $-39.77^{\circ}$ & $0.6^{\circ}$ & 0.89 & 2.06 & 8.04 & 1 & {$[11]$} \\
\hline Vela X & $-45.6^{\circ}$ & $0.8^{\circ}$ & 0.72 & 1.36 & 7 & 1 & {$[12]$} \\
\hline Vela Jr & $-46.36^{\circ}$ & $1^{\circ}$ & 1.30 & 1.87 & 4.5 & 1 & {$[13]$} \\
\hline HESSJ1614-518 (1) & $-51.82^{\circ}$ & $0.42^{\circ}$ & 0.26 & 2.42 & - & - & {$[14]$} \\
\hline HESSJ1614-518 (2) & $-51.82^{\circ}$ & $0.42^{\circ}$ & 0.51 & 2 & 3.71 & 0.5 & {$[14]$} \\
\hline Galactic Centre & $-28.87^{\circ}$ & $0.45^{\circ}$ & 0.25 & 2.3 & 85.53 & 0.5 & {$[15]$} \\
\hline \hline
\end{tabular}

Track-like events are of particular interest for the search for very localized point-like, faint sources using high statistic and good angular resolution. The results discussed here focus on tracklike events and the reconstruction specific for tracks is applied to all events. In the Letter of Intent for KM3NeT 2.0 (LoI) [3] first promising results for the point-source search using showers were presented. Preliminary studies to evaluated the cascade events expected from the RX J1713.7-3946 and the Vela X show that these events can give a contribution of about $17 \%$ to the track events.

\section{Galactic sources analysis}

The first step in the analysis is a simulation of neutrino events coming from the location in the sky of each source with the nominal extension reported by the corresponding $\gamma$-ray analysis (the source is assumed as a flat disk). The references of the $\gamma$-ray analyses used as starting point are shown in table 1. The expected neutrino flux is derived from the measured $\gamma$-ray flux using the Vissani\&Villante method described in [8], except for the RX J1713.7-3946 case discussed later. We assume sources transparent to gamma rays. The resulting neutrino flux limit will constrain the fraction of the gamma-ray flux that is of hadronic origin. Table 1 summarizes the sources considered showing their declination $\delta$, extension and the normalization constant, the spectral index and the cutoff of the assumed neutrino flux used in the following analysis. These fluxes are described by the law:

$$
\Phi_{v}(E)=\Phi_{0} E^{-\Gamma} \exp \left(-\left(E / E_{\text {cut }}\right)^{\beta}\right) \mathrm{TeV}^{-1} \mathrm{~s}^{-1} \mathrm{~cm}^{-2}
$$

where $E$ is assumed in $\mathrm{TeV}$. The fluxes in table 1 are derived assuming $100 \%$ hadronic emission.

Some of the fluxes reported need some additional clarifications. For the source RX J1713.73946 two possible neutrino fluxes are considered. One is calculated according to the Kelner prescription [9] from the $\gamma$-ray flux in [10] and indicated in the following as RX J1713.7-3946 (1), the other one is derived with the Vissani\&Villante method from the more recent HESS data in [11] and indicated as RX J1713.7-3946 (2).

For the source HESSJ1614-518, the $\gamma$-ray flux in [14] is a pure power law and the neutrino flux derived from it is a pure power law too. To test the effect of the cut-off in the spectrum, the data in the paper were fitted adding the cutoff and from this possible $\gamma$-ray flux, the neutrino flux 
was derived. Results will be shown for both fluxes, without and with cutoff, referred respectively as HESSJ1614-518 (1) and HESSJ1614-518 (2).

The Galactic Centre $\gamma$-ray flux reported in [15] is derived for two regions: a point source (PS) HESS J1745-290 centered on Sgr $\mathrm{A}^{*}$ with radius $0.1^{\circ}$ and a diffuse emission (DF) from an annulus between $0.15^{\circ}$ and $0.45^{\circ}$. In [16] Vissani et al. evaluate the neutrino spectra expected from the these two regions PS and DF. The flux considered in this paper is the sum of the PS and DF regions and the source is considered as flat disk of extension $0.45^{\circ}$.

The second step in the analysis is a multivariate analysis to distinguish 3 classes of events: atmospheric neutrinos, neutrinos coming from the source and atmospheric muons. Preliminary cuts on the reconstructed zenith angle $\theta$ and on the distance $\alpha$ between the reconstructed track and the nominal source position are applied. This is needed to speed up the algorithm and avoid that the classifier is drowned by the background. The cut on zenith angle at about $10^{\circ}$ above the horizon (adjusted for each source on the basis of the elevation reached) is needed to reduce the background of atmospheric muons. In fact neutrinos are the only particles able to traverse the whole Earth without being absorbed, so looking at upward-going events allows to select neutrinos.

The area around the source position is clearly where the ratio signal/background is more in favor of the signal. The cut applied is $\alpha<10^{\circ}$, chosen as a compromise to reduce the background without loosing the tails of the signal distribution. Even with the zenith cut, down-going atmospheric muons that are mis-reconstructed as up-going still remain in the sample. To identify this kind of events, 6 different parameters related to the quality of the reconstruction are used to train the classifier as event features. In addition to that, the other features used are $\alpha, \theta$, the numbers of hits selected during the trigger and the reconstruction procedures and the reconstructed energy. The atmospheric background is expected to have a softer spectrum than the one expected from the source, so also the energy is an important key for background discrimination.

The python-based scikit-learn package [17] is used and in particular the classifier chosen is called "ExtraTreesClassifier" (Extremely randomized trees classifier). After the training, the classifier predict the probability that each event belongs to each of the 3 considered classes. The probability that the event belongs to the "signal" class is used as probability density function (PDF) in the following step of the analysis.

To calculate the $(3 \sigma)$ discovery potential and the sensitivity, an unbinned likelihood maximization is used, similar to previous analyses [3]. The likelihood ratio is defined as

$$
L R=\sum_{i=1}^{n} \log \frac{\frac{n_{\text {sig }}}{n} \times P D F_{\text {sig }}(i)+\left(1-\frac{n_{\text {sig }}}{n}\right) \times P D F_{b k g}(i)}{P D F_{b k g}(i)}
$$

where $n$ is the total number of recorded events in a given period of time and $n_{\text {sig }}$ is the expected number of signal events in the sample of $n$ events. For each sample $L R$ is maximised as function of $n_{\text {sig }}$ and the maximum value of $L R$ is used as test statistics.

The results are shown in fig. 1: the left plot shows the discovery potential flux at $3 \sigma$ with $50 \%$ probability, $\Phi_{3 \sigma}$, and the right plot shows the sensitivity at $90 \%$ confidence level, $\Phi_{90}$, calculated according to the method in [18]. Both $\Phi_{3 \sigma}$ and $\Phi_{90}$ are divided by the normalization flux $\Phi_{0}$ of each source, so that if $\Phi_{3 \sigma} / \Phi_{0}=1$ that means that it's possible to see the source at $3 \sigma$ assuming the spectrum is $100 \%$ hadronic, if $\Phi_{3 \sigma} / \Phi_{0}=0.8$ in case is $80 \%$ hadronic and so on (assuming the 
shape of the spectrum is maintained). The sensitivity needed to see all the considered sources is reached after about 5 (7) years in the 100\% (80\%) hadronic case. For the RX J1713.7-3946, 5 years are enough to constrain the hadronic percentage at about 35\%. The observation at $3 \sigma$ requires of course more time but few years are enough to observe the RX J1713.7-3946. The Vela X has been excluded from these plots, even if it was included in the LoI [3], because its hadronic origin is very uncertain. However, the calculations done show that ARCA would have the sensitivity to see its flux in about 1.5 (3) years if it is 50\% (30\%) hadronic.
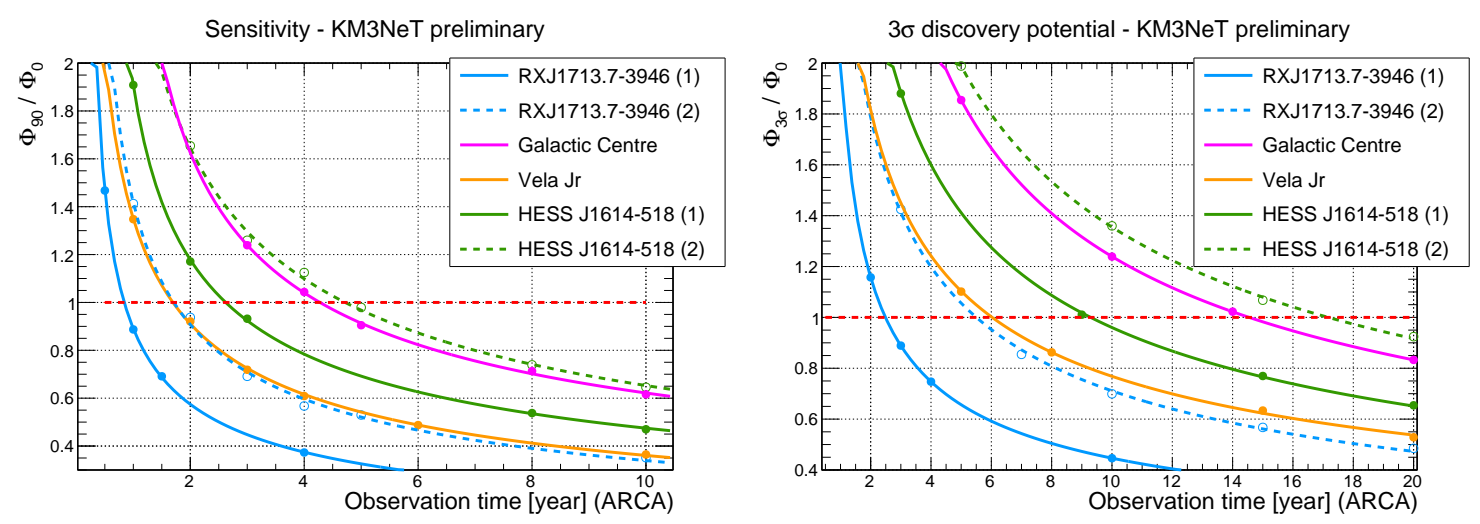

Figure 1: $\Phi_{90} / \Phi_{0}$ (left) and $\Phi_{3 \sigma} / \Phi_{0}$ (right) as function of the observation time for the sources listed in table 1.

\subsection{Stacking analysis}

Stacking different sources of the same class is a common procedure to evaluate the detectability of a specific class of sources. The sources are sorted by intensity from most to least intensity. Both the signal and background events around each source are summed maximizing the signal to noise ratio. The procedure continues until the addition of the following source becomes counterproductive because the background contribution is stronger than the signal one. Here the class of source considered is the SNR and only the two more intense sources RX J1713.7-3946 and Vela Jr have been stacked. The analysis is similar to what described previously for the single source except that the PDFs in eq. 4.2 are obtained as weighted sum of the PDFs of the single sources, both for the signal and the background. As weight, the number of events expected in each case is used. The results is shown in fig. 2 as ratio $\Phi_{3 \sigma} / \Phi_{0}$ considering the two different neutrino fluxes for the RX J1713.7-3946 discussed previously. An observation at $3 \sigma$ is possible after 2 or 3 years.

\section{Point sources with $\mathrm{E}^{-2}$ spectrum}

When no specific cosmic source spectrum is considered, typically a generic $E^{-2}$ source spectrum is assumed, as done in the recents years in the point source searches of the existent neutrino telescopes Antares and IceCube.

In this case no specific source generation is performed. Instead of having a specific training for each possible source location in the sky, only one training is performed assuming as "signal" the events distributed according to an $E^{-2}$ spectrum. Only tracks reconstructed up to $10^{\circ}$ above the 


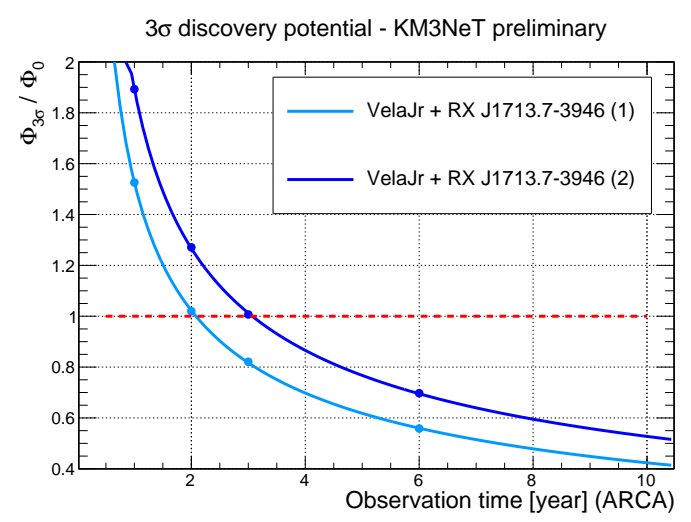

Figure 2: $\Phi_{3 \sigma} / \Phi_{0}$ as function of the observation time for the stack of RX J1713.7-3946 and Vela Jr. The neutrino fluxes of the single sources are listed in table 1.

horizon are considered. The features used for the training are the same as for the galactic source case except that the distance from the source position is not used in this case, because it is used in the subsequent likelihood analysis. The output of the training is used as variable to cut on, while the PDFs depend on the event reconstructed direction and on the reconstructed energy. The PDF used for the signal in eq. 4.2 is $P D F_{\text {sig }}(i)=\mathscr{P}\left(\psi_{i}\left(\alpha_{\mathrm{s}}, \delta_{\mathrm{s}}\right)\right) \times \mathscr{P} \mathrm{s}\left(E_{\text {rec }}^{i}\right)$ where $\mathscr{P}\left(\psi_{i}\left(\alpha_{\mathrm{s}}, \delta_{\mathrm{s}}\right)\right)$ is a parametrisation of the point spread function, i.e. the probability density function of reconstructing event $i$ at an angular distance $\psi_{i}$ from the true source location $\left(\alpha_{\mathrm{s}}, \delta_{\mathrm{s}}\right)$ and $\mathscr{P}^{\mathrm{s}}\left(E_{\mathrm{rec}}^{i}\right)$ is the probability to have a reconstructed energy $E_{\text {rec }}$ in the signal. For the background, the spatial part of the PDF depends only on the event declination $\delta_{i}$ while the probability in right ascension is uniformly distributed giving a factor $1 / 2 \pi$, so $P D F_{b k g}\left(x_{i}\right)=\mathscr{B}\left(\delta_{i}\right) / 2 \pi \times \mathscr{P}^{\mathrm{b}}\left(E_{\mathrm{rec}}^{i}\right)$.

The sensitivity and discovery flux at $5 \sigma$ as function of the source declination are shown in fig. 3 for six years of data taking. This observation time has been chosen to have a comparable exposure with respect to the IceCube results in [19].
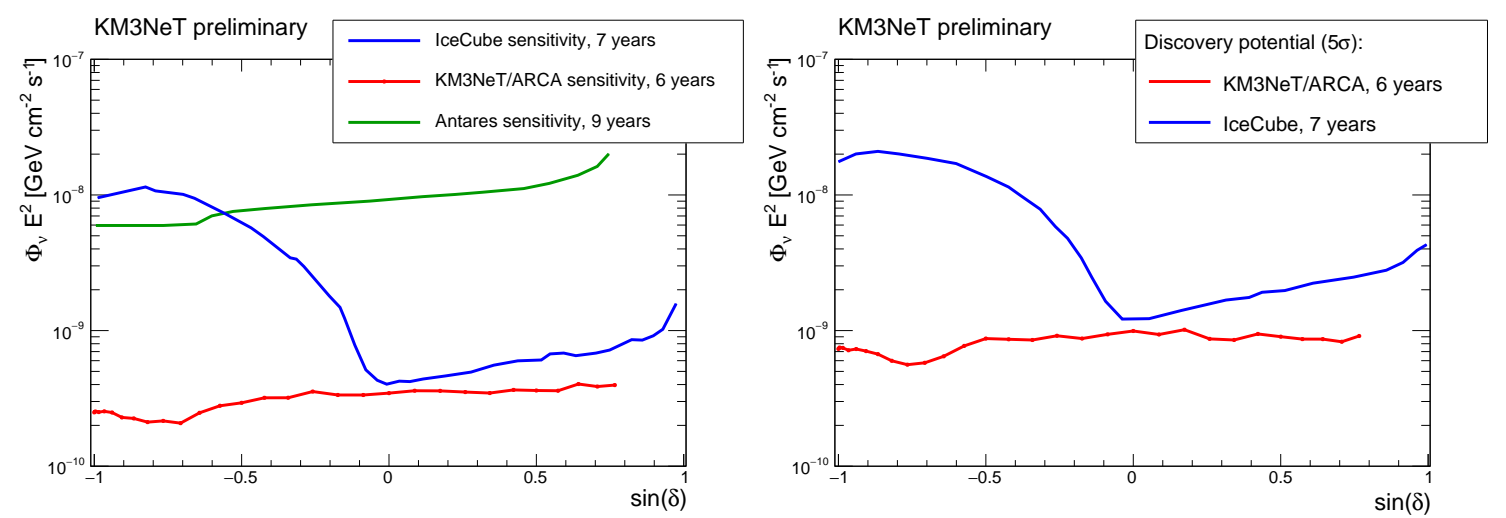

Figure 3: Sensitivity (left) and discovery flux at $5 \sigma$ (right) for a source with $\mathrm{E}^{-2}$ flux as function of the source declination. For comparison the corresponding IceCube [19] and Antares [20] fluxes are shown. 


\section{Conclusion}

Since the writing of the Letter of Intent [3] some analysis techniques have been improved: the track reconstruction discussed in [7] and the multivariate analysis allowing for the distinction of three classes of events instead that two as in [3]. The results presented show that most of the galactic sources analyzed can be observed by KM3NeT within a few years even if they are just partially hadronic. This result improves when stacking the most intense ones. The KM3NeT discovery flux and sensitivity to $E^{-2}$ flux are more than one order of magnitude better than the IceCube ones in the Southern hemisphere and comparable to it elsewhere, assuming the same exposure. In conclusion the results presented demonstrate that KM3NeT can provide important contributions to the new born field of neutrino astronomy.

\section{References}

[1] Web-site http://www.km3net.org.

[2] M. G. Aartsen et al. (IceCube Collaboration), Phys. Rev. Lett. 113 (2014), 101101.

[3] S. Adrían-Martínez et al. (KM3NeT Collaboration), J. Phys. G: Nucl. Part. Phys. 43 (2016), 084001.

[4] W. Baade and F. Zwicky, Physical Review 46 (1934) 76.

[5] D. Horns et al., A\&A, 451 (2006) L51.

[6] R. Bruijn et al. (KM3NeT Collaboration), Proc. 34th ICRC (2015), PoS(ICRC2015)1157.

[7] K. Melis et al. (KM3NeT Collaboration), Proc. 35th ICRC (2017), PoS(ICRC2017)950.

[8] F.L. Villante and F. Vissani, PRD 78 (2008) 103007; F. Vissani and F.L. Villante, NIM A588 (2008) 123; F. Vissani, Astr. Phys. 26 (2006) 310.

[9] S. R. Kelner, F. A. Aharonian and V. V. Bugayov, Phys. Rev. D 74 (2006), 063007.

[10] F. Aharonian et al. (H.E.S.S. Collaboration), A\&A 464 (2007) 235; Corrigendum in A\&A 531 (2011) C1.

[11] H. Abdalla et al. (H.E.S.S. Collaboration), arXiv:1609.08671, accepted by A\&A.

[12] A. Abramowski, et al. (H.E.S.S. Collaboration), A\&A 548 (2012), A38.

[13] H. Abdalla et al. (H.E.S.S. Collaboration), arXiv:1611.01863, accepted by A\&A.

[14] D. Gottschall et al. (H.E.S.S. Collaboration), AIP Conf. Proc. 1792 (2017) 040030

[15] A. Abramowski et al. (H.E.S.S. Collaboration), Nature 531 (2016) 476.

[16] S. Celli, A. Palladino and F. Vissani, Eur. Phys. J. C 77 (2017) 66.

[17] F. Pedregosa et al., Journal of Machine Learning Research, 12 (2011) 2825.

[18] J. Neyman, RSPTA 236 (1937), 333.

[19] M. G. Aartsen et al. (IceCube Collaboration), Astrophys. J. 835 (2017) 151.

[20] A. Albert et al. (ANTARES Collaboration), arXiv:1706.01857. 